\title{
Next-to-Next-to-Leading Order Calculation of Quasiparton Distribution Functions
}

\author{
Long-Bin Chen, ${ }^{1}$ Wei Wang $\odot,{ }^{2, *}$ and Ruilin Zhu $\odot^{3,4, \dagger}$ \\ ${ }^{1}$ School of Physics and Materials Science, Guangzhou University, Guangzhou 510006, China \\ ${ }^{2}$ INPAC, Shanghai Key Laboratory for Particle Physics and Cosmology, Key Laboratory for Particle Astrophysics and Cosmology \\ (MOE), School of Physics and Astronomy, Shanghai Jiao Tong University, Shanghai 200240, China \\ ${ }^{3}$ Department of Physics and Institute of Theoretical Physics, Nanjing Normal University, Nanjing, Jiangsu 210023, China \\ ${ }^{4}$ Nuclear Science Division, Lawrence Berkeley National Laboratory, Berkeley, California 94720, USA
}

(Received 12 July 2020; revised 18 January 2021; accepted 22 January 2021; published 19 February 2021)

\begin{abstract}
We present the next-to-next-to-leading order (NNLO) calculation of quark quasiparton distribution functions (PDFs) in the large momentum effective theory. The nontrivial factorization at this order is established explicitly and the full analytic matching coefficients between the quasidistribution and the lightcone distribution are derived. We demonstrate that the NNLO numerical contributions can improve the behavior of the extracted PDFs sizably. With the unprecedented precision study of nucleon tomography at the planned electron-ion collider, high precision lattice QCD simulations with our NNLO results implemented will enable to test the QCD theory and more precise results on the PDFs of nucleons will be obtained.
\end{abstract}

DOI: 10.1103/PhysRevLett.126.072002

Introduction.-The Feynman parton distribution functions (PDFs) are the most-important cornerstones for applying quantum chromadynamics (QCD) to high energy particle and nuclear physics. They provide not only an important platform to unveil the fundamental structure of the nucleons, but are also a crucial ingredient to explore new physics beyond the standard model at hadron colliders. Decades of extensive studies were made to probe the PDFs from hard QCD processes [1-4], while limited success was achieved from the first principle of QCD, i.e., the lattice QCD, and only a few lowest moments were obtained [5-9].

Recently the large momentum effective theory (LaMET) $[10,11]$, established to calculate various parton distribution functions directly from lattice QCD, has attracted great attention from both the phenomenology and lattice communities. Significant progress has been made, see, e.g., recent reviews [12,13] and other applications [14-16]. In LaMET, a quasidistribution is constructed from the lattice calculable matrix element of the hadron state and the relevant light-cone distributions can be derived through a perturbative matching. This provides a powerful tool to calculate all parton observables from the first principle of QCD which can be directly confronted with the experimental measurements. With the unprecedented precision study of nucleon tomography at the planned electron-ion collider (EIC) [17], high precision LaMET applications will

Published by the American Physical Society under the terms of the Creative Commons Attribution 4.0 International license. Further distribution of this work must maintain attribution to the author(s) and the published article's title, journal citation, and DOI. Funded by SCOAP ${ }^{3}$. enable us to test the QCD theory and deepen our understanding of the PDFs of a nucleon.

According to the LaMET factorization, the quasi-PDF can be expressed in terms of the light-cone PDF,

$$
\tilde{f}_{i / H}\left(y, p^{z}\right)=\int_{-1}^{1} \frac{d x}{|x|}\left[C_{i j}\left(\frac{y}{x}, \frac{|x| p^{z}}{\mu}\right) f_{j / H}(x, \mu)\right],
$$

where $\tilde{f}_{i / H}$ and $f_{j / H}$ represent the quasi-PDF and lightcone PDF, respectively, $i, j$ for the parton flavors and $\mu$ the factorization scale. In the above equation, $x \in[-1,1]$ and $y \in[-\infty, \infty]$ are the light-cone momentum and $\hat{z}-$ component momentum fractions of the hadron carried by the parton $j$ and $i$, respectively. This factorization argument is obtained on the basis that the infrared (IR) behaviors for the quasi-PDF and light-cone PDF are the same in LaMET $[10,11]$, and the matching coefficient $C_{i j}$ is perturbative calculable.

The fixed-order calculation plays an important role in the development of LaMET. It provides not only the explicit expression of the matching coefficients needed for the lattice computation, but also the detailed instances showing how the factorization works. All previous analyses are based on one-loop calculations [13]. Very recently, the twoloop studies have started, but only the ultraviolet (UV) renormalization was discussed in Ref. [18]. In this Letter, we will carry out, for the first time, the flavor nonsinglet quark distribution in LaMET at two-loop order, including the matching coefficient and the numeric improvement to extract the light-cone PDF from lattice QCD.

We emphasize two important features of our study below. First, we will demonstrate the nontrivial feature of the QCD factorization at the NNLO. Soft divergences 
will be canceled out between various contributions, whereas the collinear divergences between the quasiand light-cone PDFs cancel out. This cancellation requires the fine details of the theory, including the $\epsilon$ term and the exact scale dependence in the one-loop matching. Our explicit demonstration provides important proof of the factorization argument in LaMET [19-22].

Second, the NNLO matching results can be directly implemented in lattice calculations. As an example, we will show how this improves the previous determination of the quark PDF in LaMET. This will have a significant impact in the hadron physics community and will open new opportunities to perform high precision lattice PDF calculations in the new era [23].

To be explicit we will first derive the analytic result for the flavor nonsinglet quark distribution in LaMET at twoloop order, where various techniques developed for highorder calculations [24-27] are employed. After subtracting the UV and IR divergences, we will derive the NNLO matching coefficient into two often-used renormalization schemes. We will then show a numeric example where our new result can greatly improve the shape of the extracted quark PDFs.

LaMET factorization at two-loop order.-We will focus on the flavor nonsinglet quark distribution whose lightcone distribution follows the usual definition in the literature,

$f_{q / H}(x, \mu)=\int \frac{d \xi^{-}}{4 \pi} e^{-i x p^{+} \xi^{-}}\left\langle p\left|\bar{q}\left(\xi^{-}\right) \gamma^{+} W\left(\xi^{-}, 0\right) q(0)\right| p\right\rangle$,

where $W\left(\xi^{-}, 0\right)$ denotes the light-cone gauge link. The quark quasidistribution is defined as

$\tilde{f}_{q / H}\left(y, p^{z}\right)=\frac{p^{z}}{p^{0}} \int \frac{d z}{4 \pi} e^{i z y p^{z}}\left\langle p\left|\bar{q}(z) \gamma^{0} W(z, 0) q(0)\right| p\right\rangle$,

where the Wilson link is along the $z$ direction: $W(z, 0)=$ $\mathcal{P} \exp \left[-i g \int_{0}^{z} d z^{\prime} A^{z}\left(z^{\prime}\right)\right]$.

In the LaMET factorization of Eq. (1), both $f_{q}$ and $\tilde{f}_{q}$ contain collinear divergences. The dimensional regulation with $D=4-2 \epsilon$ and the minimal subtraction scheme ( $\overline{\mathrm{MS}}$ ) are adopted in the calculations. Equation (1) should be expanded as

$$
\begin{aligned}
\tilde{f}_{i / k}^{(2)}\left(y, \frac{p^{z}}{\mu}, \epsilon_{\mathrm{IR}}\right)= & C_{i j}^{(2)}\left(\frac{y}{x}, \frac{|x| p^{z}}{\mu}\right) \otimes f_{j / k}^{(0)}\left(x, \epsilon_{\mathrm{IR}}\right) \\
& +C_{i j}^{(1)}\left(\frac{y}{x}, \frac{|x| p^{z}}{\mu}\right) \otimes f_{j / k}^{(1)}\left(x, \epsilon_{\mathrm{IR}}\right) \\
& +C_{i j}^{(0)}\left(\frac{y}{x}, \frac{|x| p^{z}}{\mu}\right) \otimes f_{j / k}^{(2)}\left(x, \epsilon_{\mathrm{IR}}\right),
\end{aligned}
$$

where $\epsilon_{\mathrm{IR}}$ was introduced to regulate the collinear divergence and the convolution $\otimes$ integral is defined as in Eq. (1). The perturbative expansion series are collected as $T_{a}=\sum_{n=0}^{\infty}\left(\alpha_{s} / 2 \pi\right)^{n} T_{a}^{(n)}$ with $T_{a}$ being each of $\tilde{f}_{i / k}, C_{i j}, f_{j / k}$. For the flavor nonsinglet quark distribution, the collinear divergences in the light-cone PDFs $f_{j / k}^{(i)}$ on the right-hand side of Eq. (4) are known in the literature [24-27]. For the matching coefficients, the leading order is trivial: $C_{i j}^{(0)}(y)=\delta_{i j} \delta(1-y)$, and the NLO $C_{i j}^{(1)}(y)$ in $\overline{\mathrm{MS}}$ and RI-MOM schemes can also be found in Refs. [28,29].

Therefore, in order to demonstrate the factorization at NNLO, one needs to carry out the perturbative calculation of $\tilde{f}_{q}$ at two-loop order. In total, there are 79 Feynman diagrams, and three representative diagrams that are shown in Fig. 1. Virtual and real subdiagrams can be obtained by applying different cuts on the Wilson line and the total contributions satisfy vector current conservation. We shall point out that all the Feynman integrals can be classified into three families of integrals:

$$
\begin{aligned}
I_{n_{i}}^{1}= & \iint \frac{d^{D} k_{1} d^{D} k_{2}}{\left(k_{1}^{2}\right)^{n_{1}}\left(k_{2}^{2}\right)^{n_{2}}\left[\left(k_{2}-p\right)^{2}\right]^{n_{3}}\left[\left(k_{1}+k_{2}\right)^{2}\right]^{n_{4}}} \\
& \times \frac{1}{\left[\left(k_{1}+k_{2}-p\right)^{2}\right]^{n_{5}}}\left(\frac{1}{\left(P_{1}+i 0\right)^{n_{6}}}-\frac{1}{\left(P_{1}-i 0\right)^{n_{6}}}\right) \\
& \times \frac{1}{4 \pi i}\left(\frac{1}{\left(Q_{1}+i 0\right)^{n_{7}}}+\frac{1}{\left(Q_{1}-i 0\right)^{n_{7}}}\right)
\end{aligned}
$$

with $\quad P_{1}=n \cdot k_{1}+y n \cdot p, \quad Q_{1}=n \cdot k_{2} \quad$ and $n^{\mu}=\left(0, \overrightarrow{0}_{D-2}, 1\right)$;

$$
\begin{aligned}
I_{n_{i}}^{2}= & \iint \frac{d^{D} k_{1} d^{D} k_{2}}{\left(k_{1}^{2}\right)^{n_{1}}\left(k_{2}^{2}\right)^{n_{2}}\left[\left(k_{2}-p\right)^{2}\right]^{n_{3}}\left[\left(k_{1}+k_{2}\right)^{2}\right]^{n_{4}}} \\
& \times \frac{1}{\left[\left(k_{1}+k_{2}-p\right)^{2}\right]^{n_{5}}}\left(\frac{1}{\left(P_{2}+i 0\right)^{n_{6}}}-\frac{1}{\left(P_{2}-i 0\right)^{n_{6}}}\right) \\
& \times \frac{1}{4 \pi i}\left(\frac{1}{\left(Q_{2}+i 0\right)^{n_{7}}}+\frac{1}{\left(Q_{2}-i 0\right)^{n_{7}}}\right)
\end{aligned}
$$

with $P_{2}=n \cdot k_{1}+n \cdot k_{2}+y n \cdot p$ and $Q_{2}=n \cdot k_{2}$;

$$
\begin{aligned}
I_{n_{i}}^{3}= & \iint \frac{d^{D} k_{1} d^{D} k_{2}}{\left(k_{1}^{2}\right)^{n_{1}}\left(k_{2}^{2}\right)^{n_{2}}\left[\left(k_{1}-p\right)^{2}\right]^{n_{3}}\left[\left(k_{2}+p\right)^{2}\right]^{n_{4}}} \\
& \times \frac{1}{\left[\left(k_{2}-k_{1}+p\right)^{2}\right]^{n_{7}}}\left(\frac{1}{\left(P_{3}+i 0\right)^{n_{6}}}-\frac{1}{\left(P_{3}-i 0\right)^{n_{6}}}\right) \\
& \times \frac{1}{4 \pi i}\left(\frac{1}{\left(Q_{3}+i 0\right)^{n_{5}}}+\frac{1}{\left(Q_{3}-i 0\right)^{n_{5}}}\right)
\end{aligned}
$$

with $P_{3}=n \cdot k_{1}+y n \cdot p$ and $Q_{3}=n \cdot\left(k_{2}-k_{1}+p\right)$. The $+i 0$ prescription for the other propagators involving the $k_{1}, k_{2}$ is implicitly assumed. The first two families of integrals $I_{n_{i}}^{1}$ and $I_{n_{i}}^{2}$ correspond to the two kinds of cut in the left diagram in Fig. 1. The third family of integrals $I_{n_{i}}^{3}$ can 

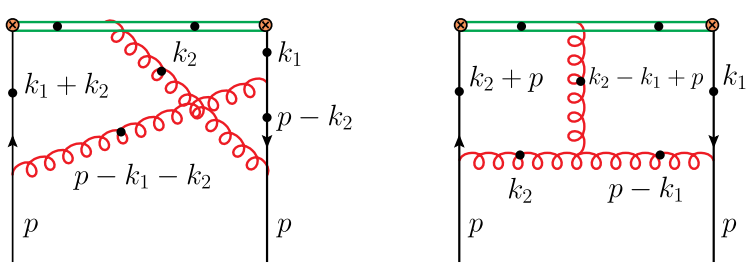

FIG. 1. Feynman diagrams for the two-loop master integrals, where the double lines correspond to the Wilson line. A dot on a propagator indicates that the power of the propagator is not always 1 and may be any integer $n_{i}$.

be obtained by the right diagram in Fig. 1. To organize the calculations of these diagrams, we use FeynRules [30] and FeynArts [31]. The algebraic manipulation and simplification of the amplitudes are performed by Mathematica packages FeynCalc [32]. We employ the integration-by-parts (IBP) techniques with the help of FIRE [33] and reduce all the involved tensor integrals into a minimal set of integrals that are called master integrals (MIs). We calculate all the MIs for both $p^{2}=0$ and $p^{2} \neq 0$ cases with the method of differential equations [34]. Inspired by Ref. [35], we construct three groups of canonical basis $\left(\mathbf{g}^{i} ; i=1,2,3\right)$ that are linear combinations of MIs [36,37], and whose differential equations can be expressed as

$$
d \mathbf{g}^{i}=\epsilon d \mathbf{M} \cdot \mathbf{g}^{i} .
$$

$\mathbf{M}$ is the matrix whose elements contain only log functions with a rational coefficient. The above form will vastly simplify the calculations. More details on the calculations of MIs can be found in Ref. [37]. These techniques developed in this calculation are also applicable to other distributions including flavor-singlet quark and gluon PDFs and generalized parton distributions.

For the UV divergences, the renormalization of the quasioperator is given as

$\tilde{f}\left(y, \frac{p^{z}}{\mu}, \epsilon_{\mathrm{IR}}\right)=\int \frac{d y_{1}}{\left|y_{1}\right|}\left[\tilde{Z}\left(\frac{y}{y_{1}}\right)\right]\left[Z^{-1} \tilde{f}\left(y_{1}, \frac{p^{z}}{\mu}, \epsilon\right)\right]$,

where $Z$ is the quark field wave function renormalization constant and $\tilde{Z}$ is the quasidistribution renormalization constant $[18,38]$. After subtracting the UV divergences, we are left with IR divergences. It contains $1 / \epsilon_{\mathrm{IR}}$ and $1 / \epsilon_{\mathrm{IR}}^{2}$ collinear divergences, and can be expressed as

$\left.\tilde{f}_{q / q}^{(2)}\left(y, \frac{p^{z}}{\mu}, \epsilon_{\mathrm{IR}}\right)\right|_{\mathrm{div}}=\frac{\Gamma_{2}^{\mathrm{IR}}(y)}{\epsilon_{\mathrm{IR}}^{2}}+\frac{\Gamma_{1}^{\mathrm{IR}}(y)+2 \Gamma_{2}^{\mathrm{IR}}(y) \log \left(\frac{\mu^{2}}{p^{z 2}}\right)}{\epsilon_{\mathrm{IR}}}$.

The explicit expressions for $\Gamma_{2}^{\mathrm{IR}}(y)$ and $\Gamma_{1}^{\mathrm{IR}}(y)$ are listed in the Supplemental Material to this Letter [39]. The $1 / \epsilon_{\mathrm{IR}}^{2}$ divergence is canceled by the last term of Eq. (4), whereas that of $1 / \epsilon_{\mathrm{IR}}$ by the last two terms. At NNLO, these divergences depend on three color structures: $C_{F}^{2}, C_{F} C_{A}$, and $C_{F} T_{F}$. The cancellations of divergences are found for all these color structures. It is necessary to emphasize that the important role of demonstrating the complete cancellation.

Matching at NNLO.-With the collinear divergence canceled out completely in Eq. (4), one can derive the matching coefficient at NNLO. In the factorization formulae of Eqs. (1) and (4), the light-cone PDF is defined in the $\overline{\mathrm{MS}}$ scheme while the matching coefficient depends on the renormalization scheme of the quasi-PDF. The regularization-independent momentum subtraction (RI-MOM) scheme is mostly adopted in lattice calculations [40], while in some quasi-PDF studies, a two-step matching procedure has been advocated in Refs. [28,41-44]. An example is the so-called modified $\overline{\mathrm{MS}}$ (MMS) renormalization scheme [44]. In this scheme, the lattice data on the quasi-PDF are firstly converted to the $\mathrm{MMS}$ scheme, and in the second step one matches the M $\overline{M S}$-renormalized quasi-PDF to the light-cone PDF. Very recently a hybrid renormalization scheme has also been proposed in Ref. [45].

The subtraction in the RI-MOM scheme can be summarized as $[29,40]$

$$
\begin{aligned}
& \left.\tilde{Z}_{\mathrm{RI}-\mathrm{MOM}}^{-1}\left\langle p\left|\bar{q}(z) \gamma^{z} W(z, 0) q(0)\right| p\right\rangle\right|_{p^{2}=-\mu_{R}^{2}, p^{z}=p_{R}^{z}} \\
& \quad=\left.\left\langle p\left|\bar{q}(z) \gamma^{z} W(z, 0) q(0)\right| p\right\rangle\right|_{\mathrm{LO}},
\end{aligned}
$$

where $\mu_{R}$ and $p_{R}^{z}$ are the two renormalization scales in the RI-MOM scheme. The corresponding matching coefficient can be written as

$$
C_{q q}^{(n), \mathrm{RI}-\mathrm{MOM}}=\left[C_{q q}^{(n), \overline{\mathrm{MS}}}\left(y, \frac{p^{z}}{\mu}\right)-\left(\tilde{f}_{q / q}^{(n)}\right)_{\text {С.T. }}\right]_{+},
$$

where $\left[C_{q q}^{(n) \overline{\mathrm{MS}}}\left(y, p^{z} / \mu\right)\right]_{+}$is the $n$ th-order matching coefficients in the $\overline{M S}$ scheme. The counterterm in the RIMOM scheme is given by

$$
\left(\tilde{f}_{q / q}^{(n)}\right)_{\text {C.T. }}=\left|\frac{p^{z}}{p_{R}^{z}}\right| \tilde{f}_{q / q}^{(n), R}\left(\frac{p^{z}}{p_{R}^{z}}(y-1)+1, \frac{\mu_{R}^{2}}{p_{R}^{z 2}}\right) .
$$

The explicit expressions for these counterterms are available in the supplementary Mathematica package files to this Letter [39].

With the factorization scale $\mu=p_{z}$, the matching coefficients in the $\overline{\mathrm{MS}}$ scheme can be decomposed into three different color structures,

$C_{q q}^{(2), \overline{\mathrm{MS}}}(y, 1)_{[i]}=\left(C_{F} c_{i}^{C_{F}}+C_{A} c_{i}^{C_{A}}+2 T_{F} n_{f} c_{i}^{T_{F}}\right) C_{F}$,

where $[i]$ represents four different kinematic regions for $y$ : $y>1,0<y<1,-1<y<0$, and $y<-1$. One interesting point is that the scale dependent single logarithm $\log \left(\mu^{2} / p^{z 2}\right)$ appears in the NNLO matching coefficients 


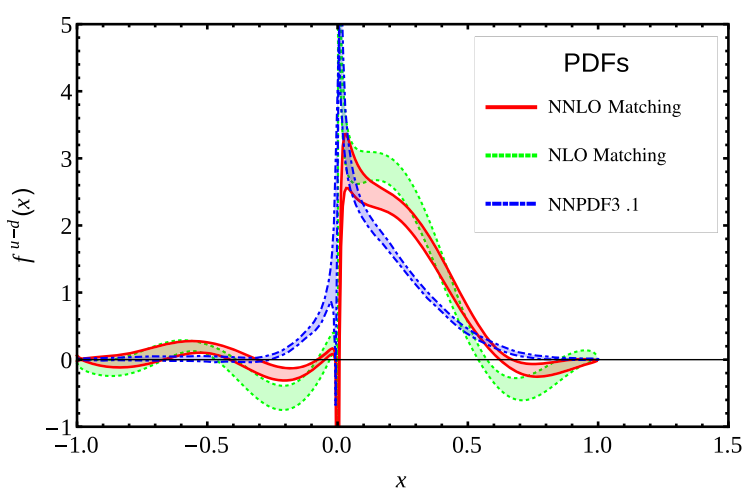

FIG. 2. Results for the light-cone PDFs $f^{u-d}(x)$ at $\mu=2 \mathrm{GeV}$ using the lattice data in the M $\overline{M S}$ renormalization scheme [44]. The result from the NNPDF3.1 global fit [2] is also shown as a comparison. An oscillatory behavior appears at NLO due to the fact that the lattice data have been truncated at $z=10 a$ in coordinate space $[44,47]$.

at all nonphysical regions. The complete expressions for $C_{q q}^{(2), \overline{\mathrm{MS}}}$ for all these regions are given in the Supplemental material to this Letter [39]. Substituting the above results into Eq. (12), one can obtain the matching coefficients in the RI-MOM scheme.

In the $\mathrm{MMS}$ scheme, the matching coefficient is obtained from $C_{q q}^{(2), \mathrm{MMS}}\left(y, p^{z} / \mu\right)$ with the asymptotic form in the $y \rightarrow \pm \infty$ region subtracted. All of the expressions of the matching coefficient $C_{q q}^{(2), \mathrm{MMS}}\left(y, p^{z} / \mu\right)$ can be found in the Supplemental Material to this Letter [39].

Numerical impact.-We adopt here the MMS renormalization scheme to demonstrate the impact of NNLO results. We use the lattice data for the M $\overline{\mathrm{MS}}$-renormalized quasi-PDF from Ref. [44]. As an example, in Fig. 2, we give the results of isovector quark distribution $f^{u-d}(x)$ extracted from the lattice data of [44] at NLO and NNLO, respectively. In the numeric calculations, we choose $\mu=$ $2 \mathrm{GeV}$ and $p^{z}=2.3 \mathrm{GeV}$. One can see from Fig. 2 that the NNLO correction is important to improve the NLO behavior and the extracted distribution at large $x$ region agrees better with the phenomenology fit from the NNPDF3.1 set [2]. An oscillatory behavior appears because the cutoff method is used and we truncate the lattice data at $z=10 a$ in coordinate space $[44,46,47]$. The NNLO corrections can soften the oscillatory behavior. We plan to have a more detailed comparison of different schemes and a detailed analysis of theoretical uncertainties in a future publication.

Conclusions.-We have for the first time explored the flavor-nonsinglet quark quasi-PDFs in the large momentum effective theory at two-loop order. With the explicit full analytic results, we found that all the collinear divergences factorized into the relevant light-cone PDFs. This has provided concrete proof of the LaMET factorization at the nontrivial two-loop order. The matching coefficient between the quark quasi-PDF and light-cone PDF was derived in the $\overline{\mathrm{MS}}$ and RI-MOM subtraction scheme. As an example, we have also shown in the M $\overline{M S}$ scheme that the NNLO corrections improve the previous lattice result for the isovector quark distribution.

We expect that more theoretical developments will follow along the direction of this Letter. In particular, the procedure and computation techniques can be extended to all other channels, including flavor singlet quark distribution and gluon distribution functions. This will complete all necessary ingredients for extracting PDFs from lattice QCD at two-loop order. Our calculation can be applied to other parton observables, such as the generalized parton distributions, transverse momentum dependent distributions, and meson distribution amplitudes. This will provide solid ground for applying lattice QCD to nucleon tomography and comparing to the experiment exploration from the future EIC.

We thank F. Yuan for all the valuable advice and discussions during the work. We thank X. Ji, Y. Ji, H.-n. Li, Y.-S. Liu, J. Wang, J. Xu, L.-L. Yang, S. Zhao, Y. Zhao, J.-H. Zhang, and Q.-A. Zhang for valuable discussions, in particular S. Zhao for discussions on the RI-MOM subtraction. We appreciate F. Yuan and $X$. Ji for reading and polishing the manuscript. We thank Z. Y. Li and Y. Q. Ma for the help in the comparison of our results with theirs. L.-B. C is supported by the National Natural Science Foundation of China (NSFC) under the Grant No. 11805042. W.W. is supported by NSFC under Grants No. 11735010, No. 11911530088 , No. 11653003, by Natural Science Foundation of Shanghai under Grant No. 15DZ2272100. R. L. Z. is supported by NSFC under Grant No. 12075124, by Natural Science Foundation of Jiangsu under Grant No. BK20171471 and Jiangsu Qing Lan Project, by China Scholarship Council under Grant No. 201906865014 and partially supported by the U.S. Department of Energy, Office of Science, Office of Nuclear Physics, under Contract No. DE-AC02-05CH11231. The numerical calculation is supported by the $\pi 2.0$ cluster supported by the Center for High Performance Computing at Shanghai Jiao Tong University.

Note added.-Recently, a preprint [48] appeared in which the authors calculated the two-loop corrections to the onshell quark correlation functions defined in the coordinate space, and the results are in agreement with ours.

* Corresponding author. wei.wang@sjtu.edu.cn Corresponding author. rlzhu@njnu.edu.cn

[1] L. A. Harland-Lang, A. D. Martin, P. Motylinski, and R. S. Thorne, Eur. Phys. J. C 75, 204 (2015). 
[2] R. D. Ball et al. (NNPDF Collaboration), Eur. Phys. J. C 77, 663 (2017).

[3] J. Gao, L. Harland-Lang, and J. Rojo, Phys. Rep. 742, 1 (2018).

[4] T. J. Hou, J. Gao, T. J. Hobbs, K. Xie, S. Dulat, M. Guzzi, J. Huston, P. Nadolsky, J. Pumplin, C. Schmidt et al., Phys. Rev. D 103, 014013 (2021).

[5] G. Martinelli and C. T. Sachrajda, Phys. Lett. B 196, 184 (1987).

[6] G. Martinelli and C. T. Sachrajda, Phys. Lett. B 217, 319 (1989).

[7] W. Detmold, W. Melnitchouk, and A. W. Thomas, Eur. Phys. J. direct 3, 13 (2001).

[8] D. Dolgov et al. (LHPC and TXL Collaborations), Phys. Rev. D 66, 034506 (2002).

[9] C. Alexandrou, S. Bacchio, M. Constantinou, P. Dimopoulos, J. Finkenrath, R. Frezzotti, K. Hadjiyiannakou, K. Jansen, B. Kostrzewa, G. Koutsou et al., Phys. Rev. D 101, 034519 (2020).

[10] X. Ji, Phys. Rev. Lett. 110, 262002 (2013).

[11] X. Ji, Sci. China Phys. Mech. Astron. 57, 1407 (2014).

[12] K. Cichy and M. Constantinou, Adv. High Energy Phys. 2019, 3036904 (2019).

[13] X. Ji, Y.S. Liu, Y. Liu, J.H. Zhang, and Y. Zhao, arXiv:2004.03543.

[14] A. V. Radyushkin, Phys. Rev. D 96, 034025 (2017).

[15] Y. Q. Ma and J. W. Qiu, Phys. Rev. D 98, 074021 (2018).

[16] Y. Q. Ma and J. W. Qiu, Phys. Rev. Lett. 120, 022003 (2018).

[17] A. Accardi, J. L. Albacete, M. Anselmino, N. Armesto, E. C. Aschenauer, A. Bacchetta, D. Boer, W. K. Brooks, T. Burton, N. B. Chang et al., Eur. Phys. J. A 52, 268 (2016).

[18] V. M. Braun, K. G. Chetyrkin, and B. A. Kniehl, J. High Energy Phys. 07 (2020) 161.

[19] J. Green, K. Jansen, and F. Steffens, Phys. Rev. Lett. 121, 022004 (2018).

[20] X. Ji, J. H. Zhang, and Y. Zhao, Phys. Rev. Lett. 120, 112001 (2018).

[21] T. Ishikawa, Y. Q. Ma, J. W. Qiu, and S. Yoshida, Phys. Rev. D 96, 094019 (2017).

[22] H. N. Li, Phys. Rev. D 94, 074036 (2016).

[23] M. Constantinou, A. Courtoy, M. A. Ebert, M. Engelhardt, T. Giani, T. Hobbs, T. J. Hou, A. Kusina, K. Kutak, J. Liang et al., arXiv:2006.08636.

[24] W. Furmanski and R. Petronzio, Phys. Lett. 97B, 437 (1980).

[25] G. Curci, W. Furmanski, and R. Petronzio, Nucl. Phys. B175, 27 (1980).
[26] S. Moch, J. A. M. Vermaseren, and A. Vogt, Nucl. Phys. B688, 101 (2004).

[27] A. Vogt, S. Moch, and J. A. M. Vermaseren, Nucl. Phys. B691, 129 (2004).

[28] T. Izubuchi, X. Ji, L. Jin, I. W. Stewart, and Y. Zhao, Phys. Rev. D 98, 056004 (2018).

[29] W. Wang, J. H. Zhang, S. Zhao, and R. Zhu, Phys. Rev. D 100, 074509 (2019).

[30] A. Alloul, N. D. Christensen, C. Degrande, C. Duhr, and B. Fuks, Comput. Phys. Commun. 185, 2250 (2014).

[31] T. Hahn, Comput. Phys. Commun. 140, 418 (2001).

[32] R. Mertig, M. Bohm, and A. Denner, Comput. Phys. Commun. 64, 345 (1991).

[33] A. V. Smirnov, Comput. Phys. Commun. 189, 182 (2015).

[34] A. V. Kotikov, Phys. Lett. B 254, 158 (1991).

[35] J. M. Henn, Phys. Rev. Lett. 110, 251601 (2013).

[36] L. B. Chen, W. Wang, and R. Zhu, Phys. Rev. D 102, 011503 (2020).

[37] L. B. Chen, W. Wang, and R. Zhu, J. High Energy Phys. 10 (2020) 079.

[38] X. Ji and J. H. Zhang, Phys. Rev. D 92, 034006 (2015).

[39] See Supplemental Material at http://link.aps.org/supplemental/ 10.1103/PhysRevLett.126.072002 for the explicit analytic expressions for the divergences of quasi-PDFs and the NNLO matching coefficients.

[40] G. Martinelli, C. Pittori, C. T. Sachrajda, M. Testa, and A. Vladikas, Nucl. Phys. B445, 81 (1995).

[41] M. Constantinou and H. Panagopoulos, Phys. Rev. D 96, 054506 (2017).

[42] C. Alexandrou, K. Cichy, M. Constantinou, K. Jansen, A. Scapellato, and F. Steffens, Phys. Rev. Lett. 121, 112001 (2018).

[43] C. Alexandrou, K. Cichy, M. Constantinou, K. Jansen, A. Scapellato, and F. Steffens, Phys. Rev. D 98, 091503 (2018).

[44] C. Alexandrou, K. Cichy, M. Constantinou, K. Hadjiyiannakou, K. Jansen, A. Scapellato, and F. Steffens, Phys. Rev. D 99, 114504 (2019).

[45] X. Ji, Y. Liu, A. Schäfer, W. Wang, Y. B. Yang, J. H. Zhang, and Y. Zhao, Nucl. Phys. B964, 115311 (2021).

[46] H. W. Lin et al. (LP3 Collaboration), Phys. Rev. D 98, 054504 (2018).

[47] Y. S. Liu et al. (Lattice Parton Collaboration), Phys. Rev. D 101, 034020 (2020).

[48] Z. Y. Li, Y. Q. Ma, and J. W. Qiu, Preceding Letter, Phys. Rev. Lett. 126, 072001 (2021). 